Methods We performed a retrospective case notes review of patients referred to pelvic radiation disease clinic over a 16 month period (Sept 2018-Jan 2020) to identify those with endoscopic evidence of RP, determine the frequency of reported symptoms, primary cancer type and treatments used for RP following referral.

Results 102 patients were seen in pelvic radiation disease clinic during the 16 month period. 54 (53\%) of these patients had endoscopic evidence of RP. Of these 54 patients, $34(63 \%)$ were male. The median age was 70 years (31-86). RP was most common in patients following prostate radiotherapy $(30,56 \%)$, followed by radiotherapy for anorectal (8, 15\%), cervical (5, 9\%), endometrial (4, $7 \%)$, vaginal $(3,6 \%)$, bladder $(1,2 \%)$ and urothelial (1, $2 \%)$ cancers, along with pseudomyxoma (1, 2\%) and Kaposi's sarcoma $(1,2 \%)$.

$23(43 \%)$ patients with RP didn't require any treatment. Of those requiring treatment, 19 (61\%) had sucralfate enemas, 18 (58\%) received endoscopically-delivered PuraStat, 1 (3\%) had hyperbaric oxygen therapy and $2(6 \%)$ were referred for radiofrequency ablation. 7 patients $(23 \%)$ needed therapy with $>1$ modality after referral.

The most commonly reported symptom of RP was rectal bleeding $(45,83 \%) .8(15 \%)$ had severe bleeding with anaemia, $28(52 \%)$ had bleeding into the toilet bowl and/or incontinence of blood and $9(17 \%)$ had bleeding on wiping. Most of the patients who developed anaemia $(7,88 \%)$ had prostate radiotherapy, 4 of whom underwent therapy with $>1$ treatment modality since re0ferral. Other commonly reported symptoms of RP included bowel urgency (17, 31\%), faecal incontinence $(18,33 \%)$ and passage of rectal mucus (7, 13\%).

Conclusions This case series suggests debilitating haemorrhagic RP is more common than previously reported. Over half of patients referred to tertiary clinic had endoscopic evidence of RP, with over half of them requiring treatment. Significant rectal haemorrhage was present in two thirds of patients and was more common following prostate radiotherapy. Those with severe rectal haemorrhage were also more likely to require $>1$ treatment modality to control their symptoms, suggesting further clinical trials are required to improve the management options for patients with haemorrhagic RP.

\section{P290 OUTCOMES OF FAECAL IMMUNOCHEMICAL TESTING FOR RISK STRATIFICATION IN A TWO-WEEK-WAIT PATHWAY FOR COLORECTAL CANCER}

${ }^{1}$ James Bailey*, ${ }^{1}$ Jill Weller, ${ }^{2}$ Caroline Chapman, ${ }^{1,3}$ Joanne Morling, ${ }^{1}$ Jonathan Alastair Simpson, ${ }^{1,3}$ David Humes, ${ }^{1}$ Ayan Banerjea. ${ }^{1}$ Nottingham University Hospitals NHS Trust, Nottingham, UK; ${ }^{2}$ Eastern Hub, Bowel Cancer Screening Programme, Nottingham, UK; ${ }^{3}$ Division of Epidemiology and Public Health - University of Nottingham, Nottingham, $U K$

\subsection{6/gutjnl-2020-bsgcampus.364}

Introduction National guidelines on the investigation of cancer recommends patients with a $3 \%$ risk or greater are investigated on an urgent cancer pathway. A review of outcomes two years after the incorporation of FIT into a 2-week-wait (2WW) pathway for colorectal cancer (CRC) was undertaken.

Methods We introduced primary care access to FIT for stratification of symptomatic patients at risk of CRC in November 2017. A retrospective review of clinical outcomes at different
FIT thresholds was undertaken. Outcomes were sourced from Cancer Outcomes and Service Dataset (COSD) to 31 December 2019.

Results 15589 FIT requests were made between November 2017 and October 2019. 90.4\% of all FIT kits dispatched were returned for analysis (13361/14788). 0.3\% of returned kits could not be analysed. FIT results $\geq 150 \mu \mathrm{g} \mathrm{Hb} / \mathrm{g}$ faeces identified patients with a $24.1 \%$ risk of CRC diagnosis (132/ 547). FIT results $100-149.9 \mu \mathrm{g} \mathrm{Hb} / \mathrm{g}$ faeces identified patients with a $12.6 \%$ risk of CRC (12.6\%). FIT results $10-99.9 \mu \mathrm{g}$ $\mathrm{Hb} / \mathrm{g}$ faeces identified a $3.6 \%$ risk of CRC (65/1829) and 4$9.9 \mu \mathrm{g} \mathrm{Hb} / \mathrm{g}$ faeces identified a $0.6 \%$ risk of CRC (10/1568). 8 CRCs were diagnosed in patients with FIT results $<4 \mu \mathrm{g}$ $\mathrm{Hb} / \mathrm{g}$ faeces out of 8921 results $(0.09 \%$ risk of CRC). Further stratification of results shows that FIT results 10-19.9 $\mu \mathrm{g} \mathrm{Hb}$ g faeces confers a $1.6 \%$ risk of CRC (11/711).

Conclusions FIT stratifies risk of CRC in a symptomatic population effectively. Risk falls below the NICE threshold for urgent investigation in some patients with $>10 \mu \mathrm{g} \mathrm{Hb} / \mathrm{g}$ faeces.

\section{P291 COLORECTAL CANCER INCIDENCE AND MORTALITY IN EUROPE. ANY CHANGE WITH THE INTRODUCTION OF SCREENING?}

Rohit Kumar*, Joe West, Timothy Card. University Of Nottingham, Nottingham, UK

\subsection{6/gutjpl-2020-bsgcampus.365}

Introduction Though there is good clinical trials evidence of the efficacy of screening for colorectal cancer (CRC), how effective it is in the real world is less clear. For an effective screening programme one would expect an initial rise in incidence before a subsequent fall, and also a fall in mortality to be observed. We therefore aimed to examine changes in incidence and mortality from CRC across Europe during the period of the rollout of CRC screening.

Methods Age-standardised CRC incidence and mortality rates per 100,000 were obtained from the European Cancer Information System (ECIS) database for 6 European countries with a CRC screening programme instituted between 2000 and 2012 and complete data for this period. Joinpoint regression analysis was used to examine the annual percentage changes in these figures and to look for changes in these trends. Full details of methodology are available in, Kim HJ, Fay MP, Feuer EJ, Midthune DN. 'Permutation

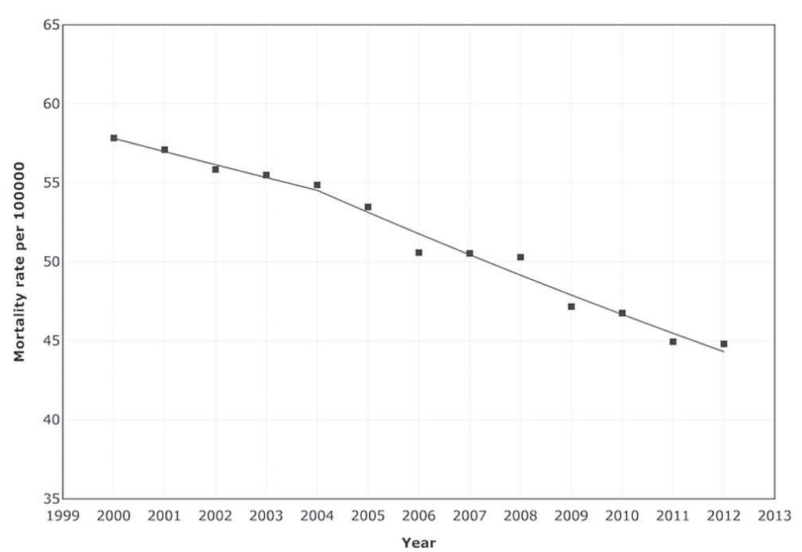

Abstract 291 Figure 1 